\title{
Instrumentos de evaluación de habilidades sociales en América Latina: un análisis bibliométrico
}

\section{Instruments to assess social skills in Latin America: A bibliometric study}

\author{
Valeria E. Morán y Fabián O. Olaz \\ Universidad Nacional de Córdoba, Córdoba, Argentina
}

\section{Resumen}

El presente estudio analizó las investigaciones que desarrollan instrumentos psicométricos para evaluar habilidades sociales y otros constructos emparentados, tales como asertividad, competencia social y autoeficacia social, en América Latina. Se analizaron 37 estudios publicados en revistas y tesis, como así también en anales y resúmenes de congresos en el periodo 1990-2013. Se estudiaron los índices de productividad y de colaboración, además de los constructos de los estudios. Los resultados indican que 2003 y 2009 fueron los años de mayor producción, y que los países más productivos fueron Brasil y Argentina. Los estudios fueron desarrollados por diversos grupos de investigación y sólo unos pocos autores han publicado más de un artículo dentro del período de estudio. Existe una tendencia a trabajar en grupos reducidos de dos o tres autores y el constructo más evaluado es habilidades sociales, especialmente en poblaciones de niños y estudiantes universitarios. Los estudios psicométricos desarrollados con mayor frecuencia incluyen el análisis de confiabilidad y validez basados en la estructura interna, utilizando análisis factorial exploratorio.

\begin{abstract}
The present study analyzed the research which develop psychometric instruments to assess social skills and related concepts such as assertiveness, social competence and social self-efficacy, in Latin America. 37 studies published in journals and theses, as well as in conference proceedings and records for the period 1990-2013 were analyzed. We studied productivity and collaboration indexes, as well as the construct of the studies. The results indicate that 2003 and 2009 were the years with the higher productivity, and that Brazil and Argentina were the most productive countries. The studies were developed by diverse groups of researchers, and only a few authors have published more than one article within the period. There is a trend to work in small groups of two or three authors, and the construct with the larger amount of research was social skills, especially among children and college students. The psychometric studies that were most frequently developed include evaluations of reliability and validity based on the internal structure, using exploratory factor analysis.
\end{abstract}

Keywords: social skills, bibliometry, assessment, Latin

\section{America.}

Palabras clave: habilidades sociales, bibliometría, evaluación, Latinoamérica. 


\section{Introducción}

El establecimiento de relaciones sociales satisfactorias que permitan a la persona desempeñarse de manera exitosa y saludable en diferentes ámbitos, tanto en los vínculos y relaciones afectivas cercanas, como en la vida profesional, laboral y académica es un factor salugénico de gran importancia. Es por ello que el estudio de las relaciones interpersonales ha sido abordado desde todas aquellas disciplinas que se ocupan del ser humano y sus espacios de actuación.

Desde un enfoque cognitivo comportamental, el repertorio de conductas que permiten al individuo relacionarse eficazmente con otras personas han sido definidas como habilidades sociales (HHSS). Estas conductas permiten manifestar actitudes, deseos, opiniones o derechos de un modo adecuado a una situación, respetando la expresión de esas conductas en los demás (Caballo, 2000).

Si bien el enfoque cognitivo comportamental ha sido uno de los modelos teóricos más prolíficos en el estudio del comportamiento interpersonal, en la actualidad, coexisten diferentes abordajes teóricos orientados a su investigación y a la intervención, los cuales utilizan una terminología diversificada para referirse a estas competencias. Así, se han propuesto diferentes constructos más o menos similares que se utilizan, no siempre correctamente, en forma intercambiable.

Desde algunos enfoques vinculados al estudio de la estructura de la personalidad se hace referencia a la existencia de ciertos factores fundamentales que serían comunes a personas de diferentes culturas, y que constituirían formas estables de comportamiento frente a diferentes situaciones. En estos modelos teóricos (tales como el Big Five Factor Model, Costa y Mc Crae, 1999) la tendencia de las personas a la sociabilidad y la facilidad para desempeñarse eficazmente en el medio social se vincula con un buen desarrollo de rasgos, como la extraversión y la amabilidad.

El funcionamiento social eficaz de una persona también es explicado por ciertas capacidades básicas para percibir, utilizar, comprender y manejar las emociones propias y las de los demás, subsumidas dentro del concepto general de Inteligencia Emocional (IE). El estudio de la IE se ha abordado desde dos modelos conceptuales: el modelo de habilidad (Mayer, Salovey y Carusso, 2000) y el modelo mixto (Goleman, 1998). Si bien ambos abordajes difieren entre sí en los constructos que se incluyen en el concepto general de IE, ambos coinciden en considerar a este conjunto de habilidades como atributos estables presentes en todas las personas.
Si bien las HHSS se vinculan conceptualmente con los constructos anteriormente revisados, los mismos han sido formulados de acuerdo a un modelo en el cual se supone que la conducta está determinada por variables o atributos personales estables presentes en todas las personas. Desde este modelo de atributo (Fernández Ballesteros, 2000) la conducta de las personas puede ser explicada atendiendo a estos rasgos, más allá de la demanda específica de una situación. Las HHSS se definen desde un modelo comportamental en el cual la explicación del comportamiento humano se fundamenta en el análisis de la conducta, el ambiente en la que esta tiene lugar, las variables personales (e.g., cogniciones, creencias, etc.) y su interacción.

Las HHSS son conductas ejecutadas en las interacciones interpersonales que permiten al individuo un desempeño social competente (Prytz Nilsson y Suarez, 2009), atendiendo a los derechos del interlocutor y de sí mismo, de una manera adecuada a la situación y al medio sociocultural en el que está inmerso, maximizando la probabilidad de refuerzo y minimizando la posibilidad de consecuencias socialmente negativas (Caballo, 1993). Gresham (2011) afirma que son comportamientos que facilitan el establecimiento y mantenimiento de relaciones sociales, contribuyen a la aceptación por parte de colegas y compañeros, y, en los niños, permiten una adaptación escolar satisfactoria.

Z. A. P. Del Prette y Del Prette (2010) indican que el concepto de HHSS debe diferenciarse del término competencia social (CP), ya que este refiere a la calidad de la conducta ejecutada según criterios de funcionalidad, es decir, de acuerdo a los efectos del desempeño social del individuo en términos de eficiencia. Desde este punto de vista, la competencia social implica la capacidad de organizar pensamientos, sentimientos y acciones en función de los objetivos y valores del individuo, como así también del escenario situacional y cultural (A. Del Prette y Del Prette, 2008; Gresham, 2011). Por otro lado, el concepto de HHSS tiene un carácter descriptivo y hace referencia al repertorio de conductas necesarias, pero no suficientes, para un desempeño social competente. En este sentido, la posesión de un adecuado repertorio de HHSS no asegura un desempeño socialmente competente, ya que solo será competente si estas habilidades son ejecutadas bajo los criterios de eficiencia mencionados. Sumado a esto, la competencia social muchas veces requiere la posesión de otros tipos de habilidades, por ejemplo, cognitivas.

Un factor que determina que la persona pueda desempeñarse en forma socialmente competente son las creencias de autoeficacia social (AS). Olaz (2012) define a la AS como las creencias que una persona tiene acerca de su capacidad para responder en forma adecuada a demandas interpersonales 
específicas, en la cual las creencias de AS tienen un papel decisivo en las interacciones sociales, ya que ayudan a organizar el conocimiento y las capacidades de ejecución en una acción social competente. Por otro lado, si bien los primeros desarrollos sobre HHSS comienzan con los estudios de la asertividad, a partir del avance en el estudio en este campo se empezó a conceptualizar la asertividad como una clase de habilidad social, dirigida a la autoafirmación y a la defensa de los derechos personales.

El estudio de las HHSS ha sido uno de los más productivos en los últimos años, debido a las implicancias que posee en diversos ámbitos, tales como el laboral, educacional, clínico y la psicología del desarrollo, entre otros. Prytz Nilsson y Suárez (2009) afirman que es posible observar una tendencia mundial de crecimiento en la producción de literatura en el área y su extensión en los últimos años a Latinoamérica. Esto queda reflejado en un mayor número de investigaciones que incluyen estudios tanto descriptivos (Contini, Coronel, Levin y Hormigo, 2012; Delevati, Lima, Milka y Silva, 2013; Matson, Hattier y Turygin, 2012; Rasmussen, Becker, McLennan, Urichuk y Andrew, 2011; Rom y Silvestre, 2012; Smith y Matson, 2010), como ex post facto (Carneiro y Falcone, 2013; Feitosa, Del Prette y Del Prette, 2012; Miers, Blöte y Westenberg, 2010; Milsom y Glanville, 2010) y estudios de eficacia de entrenamientos y programas de intervención (Caballo Escribano y Verdugo Alonso, 2005; Gantman, Kapp, Orenski y Laugeson, 2012; Gresham, Vance, Chenier y Hunter, 2013; León Rubio, León-Pérez, Herrera Sánchez y De la Corte De la Corte, 2013; Saldaña y Cruz, 2013).

Coincidiendo con Tornimbeni, Pérez y Olaz (2008), en Psicología la mayor producción científica en un área muchas veces está relacionada a la posibilidad de contar con instrumentos de evaluación para los constructos estudiados. En este sentido, la realización de investigaciones en el área de las HHSS depende en gran medida de la disponibilidad de técnicas de evaluación. Z. A. P. Del Prette y Del Prette (2011) plantean la importancia de disponer de técnicas de evaluación de las HHSS tanto para la investigación como para el ejercicio profesional, ya que los mismos son necesarios para la evaluación de las intervenciones utilizadas para el entrenamiento de estas habilidades.

Si bien las técnicas de evaluación de las HHSS son diversas (entrevistas, observación por expertos, autoobservación, pruebas de desempeño de roles, entre muchas otras), estudios bibliométricos sobre la producción científica en HHSS indican que los inventarios son una de las técnicas más empleadas (Bolsoni-Silva et al., 2006; Prytz Nilsson y Suárez, 2009; Reyna y Brussino, 2011). Esto puede deberse a que pueden ser utilizados con muestras amplias, abarcan un nú- mero grande de situaciones y comportamientos, tienen un mínimo costo y evalúan de manera objetiva pensamientos y sentimientos en situaciones sociales (Gresham y Elliot, 1984). Si bien históricamente Estados Unidos y Europa han presentado la mayor producción de investigaciones instrumentales sobre HHSS, en Latinoamérica existen grupos de trabajo que se dedican a esta tarea (Reyna y Brussino, 2011).

En este sentido, en los últimos años los estudios bibliométricos han sido cada vez más requeridos y utilizados tanto para la cuantificación de la producción como para otros fines, por ejemplo, identificar grupos y áreas de excelencia, asociaciones temáticas, interdisciplinaridad, disciplinas emergentes y redes de colaboración temática (De Filippo y Fernández, 2000). En relación a las HHSS, solo se reportan dos estudios bibliométricos sobre las producciones bibliográfica en Latinoamérica donde se evidencia un crecimiento en esta área durante la última década (Bolsoni-Silva et al., 2006; Prytz Nilsson y Suárez, 2009). Con respecto a estudios específicos sobre publicaciones instrumentales para evaluar este constructo, únicamente se reporta el trabajo de Reyna y Brussino (2011) cuyo objetivo fue analizar la producción de herramientas para medir competencia social y habilidades sociales en niños en América Latina. Las autoras revisaron las propiedades psicométricas, las dimensiones evaluadas y los países más productivos, aunque no estudiaron acabadamente indicadores bibliométricos específicos tales como productividad, colaboración y análisis de materias, considerados los más importantes para medir y cuantificar la actividad científica (Rueda-Clausen Gómez, Villa-Roel Gutíerrez y Rueda-Clausen Pinzón, 2010).

El estudio de cada uno de estos indicadores constituye un considerable aporte, no solo a la evaluación de la actividad científica en materia de psicometría en Latinoamérica, sino también se presenta como una herramienta para quienes se encuentran en la búsqueda de instrumentos de medición de las HHSS para sus estudios empíricos, o simplemente para su práctica clínica cotidiana.

Por lo señalado, el objetivo del presente trabajo es realizar un estudio bibliométrico sobre estudios de construcción y adaptación de instrumentos para evaluar HHSS, competencia social, asertividad y autoeficacia social, llevados a cabo en América Latina.

Para ello se plantea, en primer lugar, que al analizar la productividad es posible señalar qué autores y países son los más activos en la producción de instrumentos de evaluación. En segundo lugar, estudiar la colaboración aporta datos sobre la estructura social de comunidad científica dedicada al campo de las habilidades sociales, la cual puede estar cons- 
tituida por pequeños grupos y autores individuales, o bien, por grandes grupos de colaboración (Prytz Nilsson y Suárez, 2009). La importancia de este indicador radica en que un mayor nivel de colaboración se relaciona con un mayor crecimiento del campo científico del que se trate, ya que se reducen los costos económicos propios de todo proceso de investigación, se cuenta con la supervisión de pares y se facilita el acceso a material bibliográfico (López López, 1996). Finalmente, en tercer lugar, se propone realizar el análisis de materias. Este indicador permitiría establecer las tendencias en la construcción y adaptación de instrumentos de medición en el ámbito de las HHSS. De esta manera, posibilita detectar los constructos y poblaciones de interés, así como también la madurez y rigurosidad de los estudios psicométricos realizados, permitiendo identificar las limitaciones y superarlas en posteriores investigaciones. Esta dimensión analizada es de suma relevancia, ya que la psicometría corresponde a la orientación más antigua que adoptó la investigación psicológica en América Latina, y grandes aportes a esta rama a nivel global provienen de esta región (Alarcón, 1998).

\section{Método}

\section{Muestra}

Se analizaron 37 trabajos que fueron considerados, según la clasificación de Montero y León (2007), como estudios instrumentales, recabados de 34 publicaciones (identificadas con asterisco en el listado de referencias) en revistas, tesis, anales y resúmenes de congresos en el periodo 1990-2013. Se determinó este periodo debido a que el primer estudio reportado data de 1990. Con respecto al año 2013, los datos fueron recolectados hasta el mes de junio, inclusive. Se utilizaron los buscadores Dialnet, Scholar Google y se consultaron diferentes bases de datos especializadas, entre ellas, PubMed, ScienceDirect, Sage, Ebsco, Redalyc, Scielo, APA Journals, LILACS y PsicoDoc. Los descriptores empleados fueron habilidades sociales, competencia social, asertividad, autoeficacia social, y comportamiento interpersonal. Se realizaron búsquedas con cada descriptor de manera individual, como así también unidos a otros descriptores tales como evaluación, medición, escala, inventario y test. Todos los términos fueron ingresados en español, portugués e inglés. Se eliminaron artículos repetidos, artículos sobre instrumentos validados para países no latinoamericanos y artículos donde la medición de los constructos evaluados se efectuaba a través de subescalas de instrumentos más amplios.

\section{Procedimiento}

Los 37 estudios fueron codificados y ordenados para su tratamiento utilizando un diseño ex post facto retrospectivo historiográfico bibliométrico (Montero y León, 2007). Se utilizaron tres indicadores bibliométricos, considerados como relevantes para la cienciometría (Rueda-Clausen Gómez et al., 2010) y empleados en estudios bibliométricos previos sobre HHSS (Prytz Nilsson y Suárez, 2009).

El primer indicador bibliométrico analizado fue la productividad, utilizando los índices de evolución temporal (cuantificando la frecuencia de instrumentos publicados por año) y el análisis de autores y países más productivos. Para evaluar la productividad por autores se computó la cantidad de autores en la totalidad de documentos y, en segundo lugar, se registró la cantidad de veces que cada uno de ellos apareció como firmante. Con esta información se elaboró una tabla de frecuencias que permitió cuantificar la productividad de autores según el número de firmas. Finalmente, se cuantificó la cantidad de firmas de acuerdo al país de afiliación de los autores.

El segundo indicador estudiado fue la colaboración entre los autores y su evolución temporal. Para ello, se computó el número de firmas por trabajo y el número de estudios para cada número de firmas. Por otro lado, se calculó el índice de colaboración (IC) el cual permite inferir el número promedio de firmas incluidas en los artículos de cada instrumento. Finalmente, se estimó la evolución temporal del IC cada tres años (a excepción del primer intervalo, ya que desde el año 1990 al 1999 solo se analizó un estudio).

El último indicador utilizado fue el análisis de materias. Teniendo en cuenta los aspectos que son evaluados a la hora de seleccionar un test o instrumento de medición para la investigación o la práctica clínica, se cuantificaron los instrumentos de acuerdo a los constructos evaluados, las poblaciones estudiadas y los análisis psicométricos realizados, analizando específicamente el número de artículos que incluían estudios de evidencia de estructura interna, dada la importancia de esta fuente de validez para la psicometría contemporánea y para el desarrollo de modelos teóricos. Estas dimensiones han sido consideradas y analizadas en otros estudios sobre instrumentos de evaluación de HHSS (Inglés, Méndez, Hidalgo, Rosa y Estévez, 2003; Reyna y Brussino, 2011). Para el análisis de los datos y la obtención de estos indicadores se utilizaron tablas de distribución de frecuencias y estadísticos descriptivos tales como porcentajes y frecuencias.

\section{Resultados}

\section{Productividad}

A partir de los resultados obtenidos se observó que el primer estudio publicado sobre el tema fue en 1990, no registrán- 
dose trabajos publicados con anterioridad a esa fecha. Otro dato importante es que se registra un lapso de nueve años entre el primer y el segundo estudio. Es recién a partir del año 2001 cuando la producción se muestra continua, y con un mayor número de publicaciones respecto a los años anteriores. A partir de esa fecha el año más productivo fue el 2009 con nueve publicaciones. En el 2010 y posteriores se registra una disminución de trabajos con respecto al 2009. Esto puede ser apreciado en la figura 1.

Se observó que de un total de 71 autores, 59 de ellos firmaron solamente un trabajo (83.1\%). Luego, se visualizó un $8.45 \%$ de autores con dos firmas, y un $5.63 \%$ de autores con tres firmas. Solamente el $2.82 \%$ de los autores firmó más de ocho estudios.

Con los datos recabados se identificaron a los 12 autores con un alto número de publicaciones sobre instrumentos de evaluación de HHSS y constructos emparentados en América Latina. Entre ellos, los más productivos son: Zilda Del Prette con 11 estudios, seguido por Almir Del Prette con ocho trabajos y Reyna, Bolsoni-Silva, Marturano, y Olaz, con tres. Es destacable que los dos autores más productivos firman más del doble de trabajos que los autores subsiguientes.

Para determinar los países más productivos se consideró la afiliación institucional de cada autor. Se observó que el país más productivo es Brasil, ya que cuenta con 19 estudios de un total de 37 , representando el $48.7 \%$ de los trabajos. El segundo país más productivo es Argentina con 11 estudios, es decir, el 28.2\% del total. México se ubica en tercer lugar con cinco estudios (12.8\%) y Colombia cuarto, con dos estudios (5.1\%). Costa Rica y Chile representan el 5.2\% de lo publicado con un estudio cada uno. Estos resultados coinciden con las afiliaciones de los 12 autores con mayor cantidad de trabajos, donde siete de ellos pertenecen a universidades de Brasil, cuatro pertenecen a una universidad de Argentina y solo uno a una universidad de México.

Finalmente, es importante destacar que de los 21 países que integran América Latina solamente seis han publicado estudios instrumentales sobre HHSS en el periodo estudiado.

\section{Colaboración}

Se detectó que seis trabajos han sido firmados por un autor y los 31 restantes fueron realizados en coautoría. La mayor parte de estos estudios son firmados por dos, tres y cuatro autores, representando casi el $78.3 \%$ de los trabajos. Por otro lado, solo dos estudios fueron firmados por más de cuatro autores.

El índice de colaboración obtenido a partir de 102 firmas sobre 37 trabajos fue de 2.76. Esto indica que el número promedio de investigadores que firman o colaboran por trabajo es cercano a tres personas. En la figura 2 puede observarse la evolución temporal de dicho índice, la cual permite inferir una progresiva y fuerte tendencia a la colaboración y al trabajo en equipos de investigación.

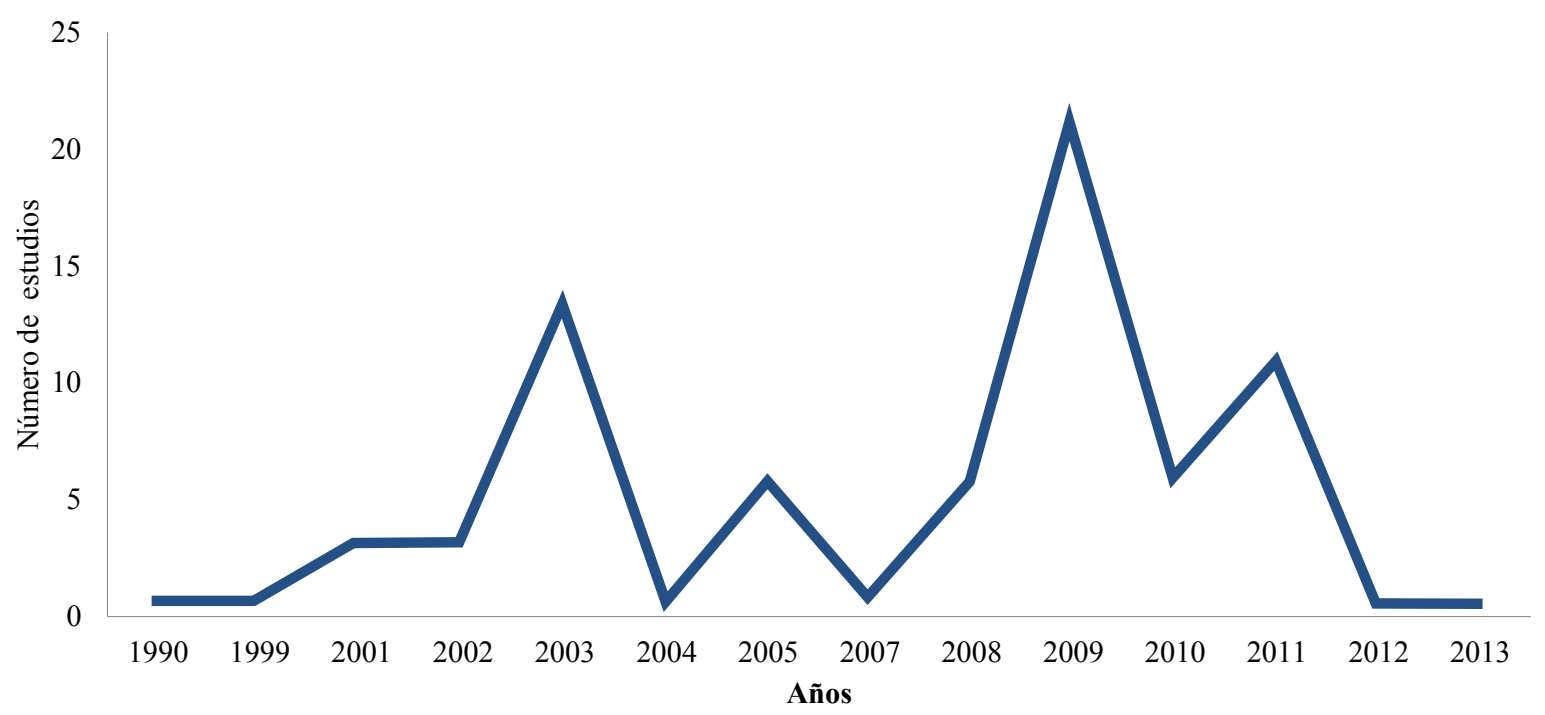

Figura 1. Evolución temporal de la productividad. Período 1990-2013. 


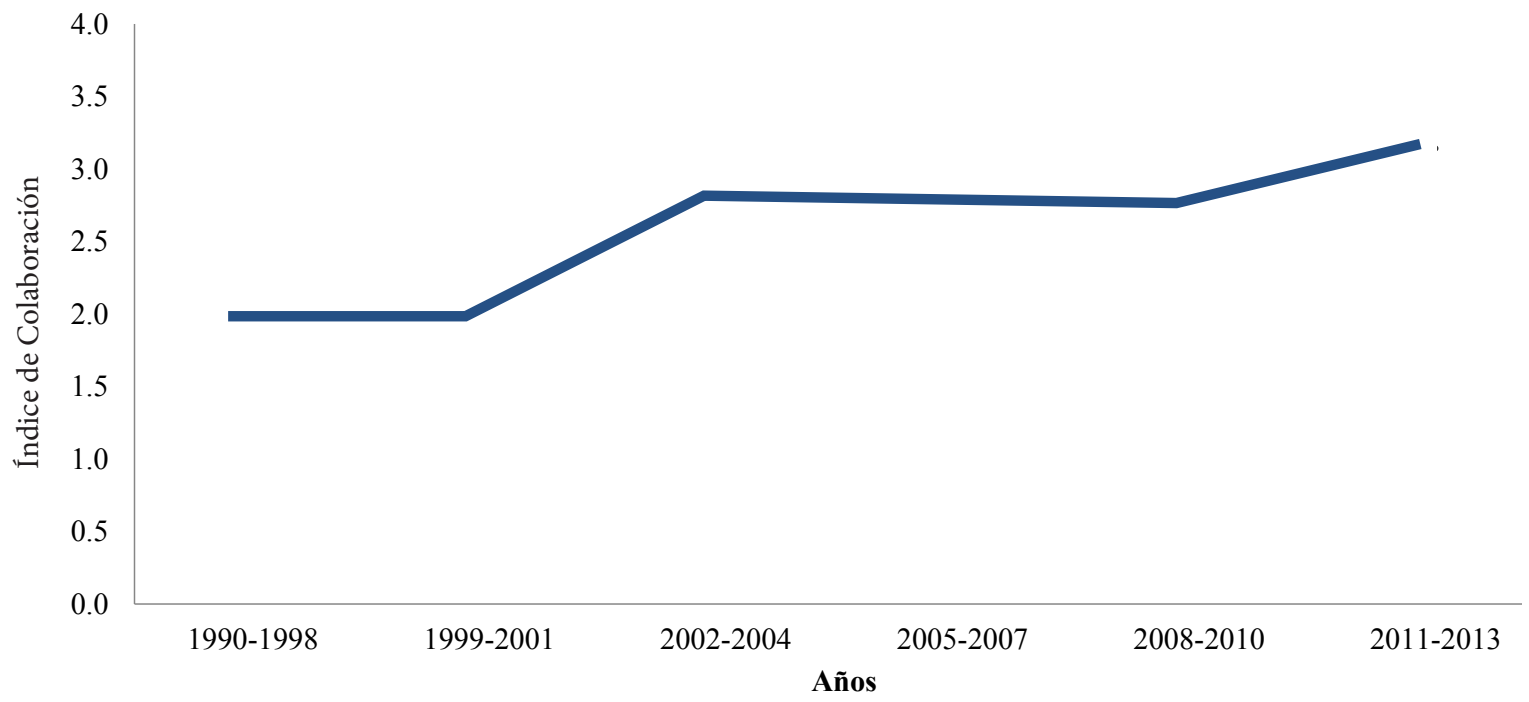

Figura 2. Evolución temporal del Índice de Colaboración cada 3 años.

\section{Análisis de materias}

El constructo más evaluado en el periodo 1990-2013 ha sido Habilidades Sociales, representando el 59.46\% de los trabajos. Con un valor significativamente inferior se encuentran competencia social y asertividad (18.92\%), seguidas de autoeficacia social (2.7\%). Los resultados se exponen en la figura 3.
El mayor número de instrumentos evalúa población infantil (19 trabajos), representando el 51.35\% del total. En segundo lugar, se observan siete instrumentos de evaluación para estudiantes universitarios y cuatro para adolescentes. Las poblaciones menos estudiadas son pacientes psiquiátricos y parejas, con un instrumento, representando solamente un $5.4 \%$ del total (ver figura 4 ).

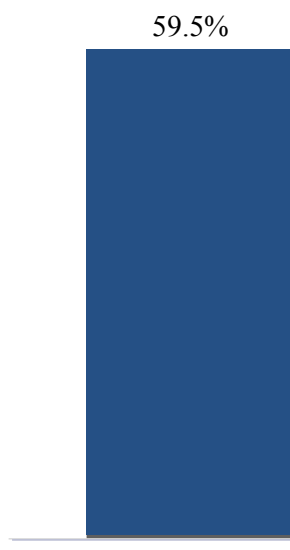

HHSS

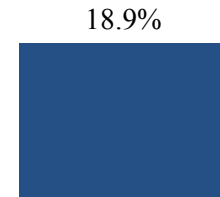

Competencia Social

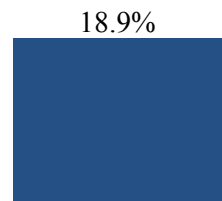

Asertividad
$2.7 \%$

Autoeficacia Social

Figura 3. Constructo evaluado según número de estudios. 


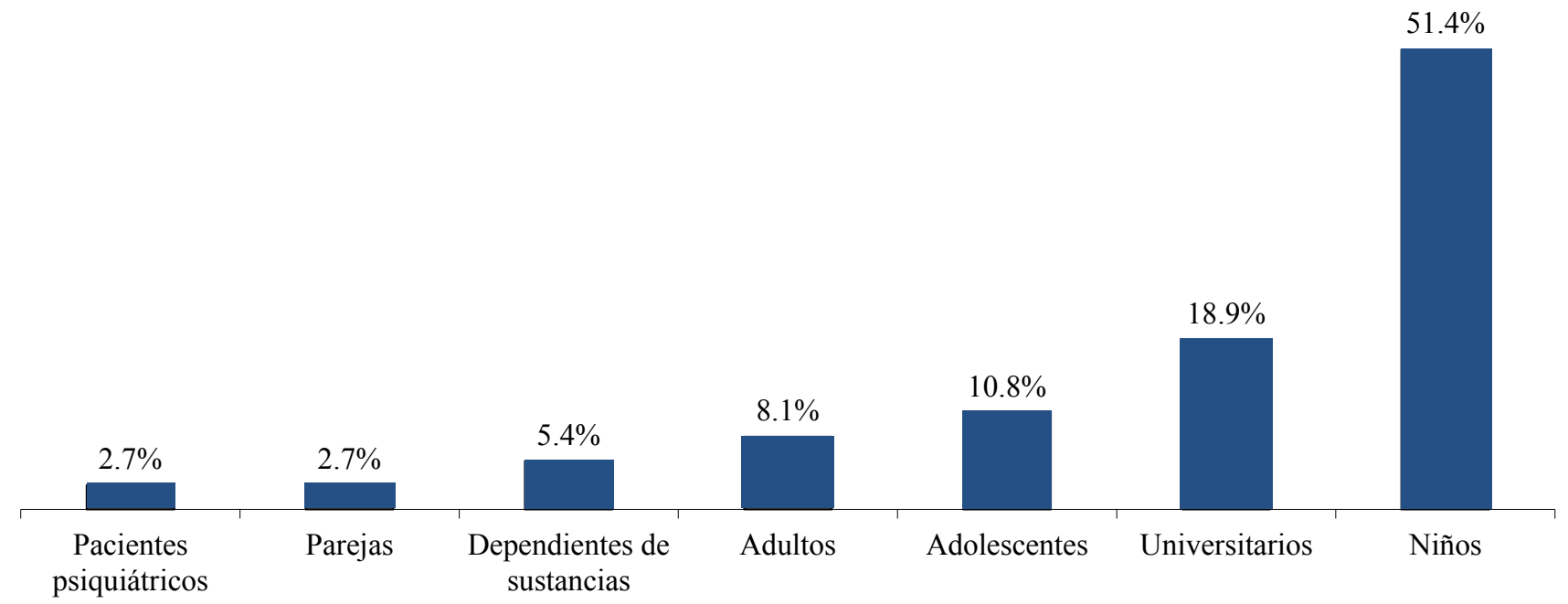

Poblaciones estudiadas

Figura 4. Población evaluada según número de estudios.

\section{Características de los instrumentos utilizados}

En relación a la modalidad de administración de los instrumentos, se observa que el autoinforme es la modalidad empleada en 23 estudios (62.16\%), y el heteroinforme se utiliza en $14(37.84 \%)$.

Los objetivos de los estudios psicométricos realizados en el período se distribuyeron de forma proporcional, ya que un poco más de la mitad de los trabajos consistió en estudios de construcción de instrumentos (57\%), mientras que el resto estuvo constituido por estudios de adaptación de instrumentos construidos para otros países o poblaciones (43\%).

Con respecto a las propiedades psicométricas analizadas, se encontró que en el $81.08 \%$ de los estudios evaluó la confiabilidad y aportó evidencia de validez de los instrumentos. Por otro lado, en un $10.81 \%$ de los trabajos solo se analizó la confiabilidad.

También se observó que en un $8.11 \%$ de los trabajos no se analizó ninguna propiedad psicométrica; en esta categoría se incluyeron informes que en sus publicaciones no presentaban ninguno de los estudios psicométricos recomendados por las normas de AERA-APA-NCME (1999) y sugeridos por Carretero-Dios y Pérez (2005). Por el contrario, estos trabajos presentaban el proceso de diseño de ítems y sus fundamentos teóricos, creación de baremos o normas de inter- pretación, así como análisis estadísticos descriptivos sobre el constructo en la población meta para la que se construyó el instrumento.

Con respecto al análisis de validez mediante evidencia de estructura interna, los resultados indican que el 64.86\% (24 trabajos) realizó análisis factorial exploratorio (AFE), el 8.11\% realizó análisis factorial confirmatorio y el 27.03\% (10 trabajos) no aportaron evidencias sobre la estructura interna del instrumento.

\section{Discusión}

El estudio de las HHSS es un área de especial interés dada la importancia de estas habilidades para la prevención de diferentes trastornos (Parault, Davis y Pellegrini, 2007; Penn, Kohlmaier y Corrigan, 2000; Troisi, Spalletta y Pasini, 1998) y para el logro de un adecuado bienestar psicológico (Bermúdez, Álvarez y Sánchez, 2003; Brackett, Rivers, Shiffman, Lerner y Salovey, 2006). Este campo de estudio se ha desarrollado y complejizado conforme al avance de las ciencias sociales y la creciente demanda e interés por parte de aquellos ámbitos donde las relaciones interpersonales tienen un papel crucial. Por esto mismo, la producción de conocimientos, la investigación empírica y el diseño de programas de intervención y entrenamiento en HHSS eficaces requieren de dispositivos de medición y evaluación que se ajusten y adapten a los contextos de análisis y aplicación actuales. En base a 
estas consideraciones, el objetivo de este trabajo fue realizar un estudio bibliométrico sobre los instrumentos construidos y adaptados para evaluar habilidades sociales, competencia social, asertividad y autoeficacia social, en América Latina.

Respecto a la evolución temporal de la productividad se encontró que el primer instrumento de evaluación de HHSS se reporta en el año 1990, y a partir de allí hubo un importante descenso de publicaciones hasta 1999, donde se comienza a visualizar una producción científica activa en materia de estudios instrumentales. Si bien se observa un crecimiento en el área, la tendencia no es constante, ya que se observaron picos notables de producción en los años 2003 y 2009. La marcada disminución a partir de 2010 podría explicarse por la posibilidad de que existan artículos o trabajos que aún no hayan sido publicados, tomando en consideración el extenso tiempo que caracteriza a los procesos editoriales de las revistas científicas de idioma español, previos a la publicación (Russell, 2004).

En relación a la productividad de los autores, se observó que la mayor productividad se concentra en dos autores, Zilda Del Prette y Almir Del Prette, quienes son considerados los más representativos del área de las HHSS en Latinoamérica, según estudios bibliométricos anteriores (Bolsoni-Silva et al., 2006; Prytz Nilsson y Suárez, 2009; Reyna y Brussino, 2011). Estos autores y su grupo de investigación tienen filiación en universidades de Brasil, el país con mayor productividad en estudios instrumentales, seguido por Argentina, tendencia ya evidenciada por Reyna y Brussino (2011) en un estudio similar sobre instrumentos de evaluación de HHSS en niños, y por Prytz Nilsson y Suárez (2009) en su estudio bibliométrico sobre HHSS.

En relación a los resultados obtenidos en el análisis de la colaboración puede observarse que la mayor parte de los estudios es firmado por dos, tres y cuatro autores, y que esta tendencia se mantiene a lo largo del periodo analizado, lo cual es consistente con lo reportado por Prytz Nilsson y Suárez (2009). Esto evidencia la tendencia paulatina a la conformación de equipos de trabajo y grupos de investigación, lo cual puede ser un indicador de una mayor "madurez" de la investigación en el área (López López, 1996).

En referencia al análisis de materias, se encontró que el constructo más evaluado son las HHSS, seguidas por, la competencia social y la asertividad. Estos datos evidencian la complejización del área de investigación de las HHSS como un constructo de mayor amplitud que el de asertividad, y diferenciado del de competencia social. En este punto es importante aclarar que el presente estudio consideró los constructos tal y como los autores los definen en sus publicaciones, sin profundizar en las definiciones conceptuales sobre las cuales se basan, lo que puede traer aparejado que en algunos trabajos se esté empleando los conceptos competencia social, asertividad y habilidades sociales como sinónimos. Por esto, es recomendable realizar estudios posteriores en donde se pueda considerar este aspecto en el análisis documental.

Otro dato importante en este análisis fue que solo se registró un instrumento que evalúa autoeficacia social, lo cual puede explicarse atendiendo a que los aportes de la Teoría Social Cognitiva (TSC) al campo de las HHSS es una área de desarrollo reciente (Olaz, 2011, 2012). En los últimos años, la TSC de Albert Bandura se ha trasformado en uno de los movimientos psicológicos más prominentes de la época. Esta teoría constituye un intento de abordaje del funcionamiento humano que revaloriza el papel de las autocreencias en la motivación y conducta humana, enfatizando el rol proactivo y autorregulador de la persona, en lugar de otorgarle un papel reactivo a fuerzas externas o impulsos biológicos. Aun cuando esta teoría es de gran impacto a nivel internacional, en Latinoamérica el estudio de las HHSS desde ella es aún incipiente

Respecto a las poblaciones evaluadas por los instrumentos, la evaluación de HHSS en población infantil fue la que presenta una mayor frecuencia (51.35\%). Reyna y Brussino (2011) indican que uno de los motivos del incremento en técnicas de evaluación del comportamiento interpersonal en la infancia se debe a las repercusiones que dicho comportamiento tiene, no solo en las edades tempranas, sino también en el desempeño futuro, y se constituye así como una oportunidad para la prevención e intervención. El segundo grupo más evaluado son los universitarios, lo cual coincide con el mayor interés observado en estos últimos años por el estudio de competencias profesionales. En este sentido, en líneas de trabajo recientes se señala a la etapa de formación superior como crucial en la adquisición y fortalecimiento de HHSS para la vida profesional, fundamentalmente en carreras donde las relaciones interpersonales forman parte del campo de trabajo, tal es el caso de Psicología (Z. A. P Del Prette y Del Prette, 2003b; Herrera Lestussi, Freytes, López y Olaz, 2012; Olaz, Cabanillas y Medrano, 2011).

Finalmente, respecto a las características de los instrumentos se encontró que están disponibles instrumentos de autoinforme y heteroinforme en iguales proporciones, esto indica una ventaja a la hora de realizar una evaluación del constructo, dada la especificidad contextual y la multidimensionalidad del mismo. Por otro lado, puede observarse en los resultados que en un $81.08 \%$ de los estudios se reportan estudios de confiabilidad y validez. No obstante, es importante indicar que un muy bajo porcentaje de estos estudios inclu- 
yen estudios que aporten evidencias de estructura interna utilizando análisis factorial confirmatorio. Este punto requiere una especial consideración ya que el establecimiento de modelos teóricos claros y con evidencia empírica acerca del número de clases de respuesta propuestas como parte del concepto de HHSS es quizás una de las mayores dificultades observadas en este campo de estudio.

Coincidiendo con Olaz (2012), en general los investigadores del área han utilizado el AFE para el establecimiento de factores y han considerado que la evidencia obtenida por medio de este procedimiento es un fundamento suficiente para ello. Según Richaud (2005), la aplicación empírica del AFE se utiliza muchas veces con el objetivo de identificar constructos o factores subyacentes por medio del estudio de la covarianza entre variables observadas, sin embargo, su empleo puede conducir al investigador a la identificación de estructuras de carácter empírico, dependientes de las muestras, y no replicables con facilidad (Tornimbeni et al., 2008). Carretero Dios y Pérez (2005) señalan que el análisis factorial agrupa diferentes variables de acuerdo a las correlaciones que se observan, aunque esta agrupación pueda deberse a circunstancias ajenas a lo conceptual. Por esto, consideramos fundamental la realización de estudios que aporten evidencia de estructura interna utilizando técnicas más robustas como el análisis factorial confirmatorio; sorprende que su utilización sea aún incipiente en los estudios instrumentales reportados.

Resulta aún más llamativo que un $8.11 \%$ de los artículos presenta instrumentos de evaluación sin analizar las propiedades psicométricas fundamentales para garantizar la cientificidad de los resultados obtenidos (AERA-APA-NCME, 1999; Carretero Dios y Pérez, 2005). En este sentido, es preocupante que las revistas, los congresos y las universidades, como espacios de divulgación científica avalen estas prácticas mediante su publicación. Dada la importancia que adquiere la utilización de instrumentos con adecuadas propiedades psicométricas tanto en la investigación como en el ejercicio profesional, se considera que los resultados de esta investigación pueden servir como fundamento para la elaboración de políticas científicas adecuadas a las necesidades de la población de la región.

Los indicadores bibliométricos constituyen una de las herramientas más utilizadas para la medición del producto de la investigación científica, ya que los mismos aportan datos sobre la estructura social de una determinada comunidad científica. Por esto mismo, en los países desarrollados estos indicadores se han consolidado como instrumentos de apoyo a los evaluadores y gestores de la política científica y tecnológica, conjuntamente con otros indicadores. Tal como señalan Prytz Nilsson y Suárez (2009), estas políticas no se conciben sin el análisis de la productividad, ya que facilita conocer el resultado global de la literatura científica en un nivel dado de especialización. Asimismo, se coincide con estos autores al considerar que los indicadores bibliométricos permiten identificar las tendencias en investigación y en qué medida estas responden a las necesidades de un país y una región.

En relación a esto, aun cuando se observa una mayor productividad en el campo de las HHSS en la región, podemos concluir que la productividad científica dista mucho a la observada en países más desarrollados y con mayor inversión en ciencia. Quizás por esto, el inicio de la producción en el área pueda observarse veinte años después que en el resto del mundo, ya que es a partir de 1970 que se observa una gran producción de publicaciones sobre HHSS a nivel internacional (Prytz Nilsson y Suárez, 2009). Asimismo, si bien se observa una tendencia al trabajo en colaboración entre autores de la región, esta tendencia dista mucho de la tendencia observada en países desarrollados, y es más clara en los países más productivos, en este caso Brasil y Argentina. Es importante señalar que puede observarse trabajos en colaboración entre autores de estos dos países, pero esto no ocurre con el resto de los países. Tal como señala Russell (2004) parece observarse aún que en la mayor parte de la región, el conjunto de entidades científicas y tecnológicas se presenta como una red desarticulada, y los vínculos tienden a ser débiles, de forma tal que se carece de estrategias de coordinación que sirvan de orientación en el ejercicio de producir conocimiento.

Por esto, los resultados observados en este trabajo pueden tomarse como un punto de partida para identificar las principales tendencias de investigación y así enriquecer la discusión conceptual vinculada a la temática. Aun así, permiten asumir que el crecimiento de la producción a nivel teórico y de investigación empírica en Latinoamérica sobre las HHSS reportado en otros estudios también se acompaña de un desarrollo paralelo en instrumentos de evaluación. De la misma manera, la producción se concentra en países donde la inversión en investigación psicológica y el trabajo en colaboración es mayor, tal es el caso de Brasil.

Para finalizar, es importante señalar la importancia de investigaciones futuras que permitan complementar los datos aquí expuestos, estudiando la productividad de las revistas, como así también los colegios invisibles y universidades más productivas. Sumado a esto, se considera fundamental la realización de un estudio que permita la comparación de indicadores bibliométricos entre América Latina y otras regiones del mundo. 


\section{Referencias}

* Las referencias marcadas con un asterisco indican los estudios incluidos como muestra en el análisis.

AERA-APA-NCME (1999). Standards for educational and psychological testing. Washington, DC: American Psychological Association, American Educational Research Association y National Council on Measurement in Education.

Alarcón, R. (1998). La investigación psicológica en América Latina: un enfoque histórico. Revista de Psicología de la Universidad Nacional Mayor de San Marcos, 2(1). Recuperado de http:// sisbib.unmsm.edu.pe/BVRevistas/psicologia/1998_n1/ investg.htm

*Álvarez Ramírez, L. Y., Saldaña, C., Muñoz Ardila, J. A. y Portela Meza, V. (2011). Asertividad, escucha y afrontamiento de la crítica en adolescentes en situación de calle. Revista Latinoamericana de Ciencias Sociales, Niñez y Juventud, 7(2) 1535-1543.

*Bandeira, M. (2002). Escala de Avaliação da Competência Social de Pacientes Psiquiátricos através de Desempenho de Papéis: EACS. Avaliação Psicológica, 1(2), 159-171.

*Bandeira, M., Del Prette, Z. A. P., Del Prette, A. y Magalhães, T. (2009). Validação das escalas de habilidades sociais, comportamentos problemáticos e competência acadêmica (SSRS-BR) para o ensino fundamental. Psicologia: teoria $e$ pesquisa, 25(2), 271-282.

*Bartholomeu, D., Silva, M. C. R. D. y Montiel, J. M. (2011). Teste de habilidades sociais para crianças: evidências psicométricas de uma versão inicial. Psico Universidade de São Francisco, 16(1), 33-43.

Bermúdez, M. P., Álvarez, I. T. y Sánchez, A. (2003). Análisis de la relación entre inteligencia emocional, estabilidad emocional y bienestar psicológico. Universitas Psychologica, 2(1), 27-32.

*Bermúdez Jaimes, M. E. (2010). Diseño, construcción y análisis psicométrico de una escala de competencia social para niños de 3 a 6 años. Versión padres de familia. Revista Iberoamericana de Psicología: Ciencia y Tecnología, 3(1), 49-65.

*Bolsoni-Silva, A. T. (2008). Roteiro de Entrevista de Habilidades Sociais Educativas Parentais (RE-HSE-P): Categorias e testagem preliminares. En L. D. Weber (Ed.), Família $e$ Desenvolvimento - Visões Interdisciplinares (pp. 145-158). Curitiba: Juruá.

Bolsoni- Silva, A. T., Del Prette, Z., Del Prette, G., Montagner, A. R., Bandeira, M. y Del Prette, A. (2006). Habilidades sociais no Brasil: uma análise dos estudos publicados em periódicos. En M. Bandeira, Z. A. P. Del Prette y A. Del Prette (Eds.), Estudos sobre habilidades sociais e relacionamento interpessoal (pp. 1-45). Sao Paulo: Casa do Psicólogo.

*Bolsoni-Silva, A. T., Marturano, E. M. y Loureiro, S. R. (2009). Construction and validation of the brazilian Questionario de Respostas Socialmente Habilidosas segundo relato de professores (QRSH-PR). Spanish Journal of Psychology, 12(1), 349-359.
${ }^{\star}$ Bolsoni-Silva, A. T., Marturano, E. M. y Loureiro, S. R. (2011). Estudos de Confiabilidade e Validade do Questionário de Respostas Socialmente Habilidosas Versão para Pais-QRSHPais. Psicologia: Reflexão e Crítica, 24(2).

Brackett, M. A., Rivers, S. E., Shiffman, S., Lerner, N. y Salovey, P. (2006). Relating emotional abilities to social functioning: A comparison of self-report and performance measures of emotional intelligence. Journal of personality and social psychology, 91(4), 780-795. doi: 10.1037/00223514.91.4.780

Caballo, V. E. (2000). Manual de evaluación y entrenamiento de las habilidades sociales. Madrid: Siglo XXI.

Caballo Escribano, C. y Verdugo Alonso, M. A. (2005). Habilidades sociales. Programa para mejorar las relaciones sociales entre niños $y$ jóvenes con deficiencia visual y sus iguales sin discapacidad. Madrid: Organización Nacional de Ciegos Españoles.

${ }^{\star}$ Cadoche, L., Frank, F. y Henzenn, H. (2009). Instrumento para la evaluación de habilidades sociales. En I. Zapico y S. Tajeyan (Eds.), Actas de la VII Conferencia Argentina de Educación Matemática (pp. 388-392). Buenos Aires: Sociedad Argentina de Educación Matemática.

Carretero Dios, H. y Pérez, C. (2005). Normas para el desarrollo y revisión de estudios instrumentales. International Journal of Clinical and Health Psychology, 5(3), 521-551.

Carneiro, R. S. y Falcone, E. M. D. O. (2013). O desenvolvimento das habilidades sociais em idosos e sua relação na satisfação com a vida. Estudos de Psicologia, 18(3), 517-526.

*Castillo, R. D., Pérez-Salas, C. P., Bravo, C., Cancino, M. G., Catalán, J. y Acosta, H. C. (2008). Diseño y validación de una Escala de Competencia Comunicativa y Social para niños. Terapia psicológica, 26(2), 173-180.

${ }^{\star}$ Cattan, A. (2005). Estudio comparativo de autoestima y habilidades sociales en pacientes adictos de una comunidad terapéutica. Salud y drogas, 5(1), 139-164.

${ }^{*}$ Cervantes, S. C., Lagunes, I. R., Rivera Aragón, S. y Díaz Loving, R. (2011). Estandarización del Inventario de Asertividad de Gambrill y Richey-II. Revista Iberoamericana de Diagnóstico y Evaluación Psicológica, 29(1), 27-50.

*Chávez, M. (2001). Elaboración de un instrumento con validez de contenido y constructo para la evaluación de habilidades sociales en niños (Tesis de maestría inédita). Facultad de Estudios Superiores Iztacala, Universidad Nacional Autónoma de México, México.

Contini, N., Coronel, C. P., Levin, M. y Hormigo, K. (2012). Las habilidades sociales en contextos de pobreza. Un estudio preliminar con adolescentes de la Provincia de Tucumán. Perspectivas en Psicología, 7(1), 112-120.

Costa, P. y Mc Crae, R. (1999). NEO-PI-R. Manual. Madrid: TEA ediciones.

De Filippo, D. y Fernández, M. T. (2000). Bibliometría: importancia de los indicadores bibliométricos. Red Iberoamericana de Indicadores de Ciencia y Tecnología, 69-76. Recuperado de http//:www.ricyt.edu.ar/interior/difusion/pubs/elc/10.pdf 
Del Prette, A. y Del Prette, Z. A. P. (2008). Psicologia das relações interpessoais e habilidades sociais: vivências para o trabalho em grupo (6ta Ed.). Petrópolis: Vozes.

*Del Prette, Z. A. P. y Del Prette, A. (2001). Inventário de Habilidades Sociais (IHS-Del-Prette): manual de aplicação, apuração e interpretação. São Paulo: Casa do Psicólogo.

${ }^{*}$ Del Prette, Z. A. P. y Del Prette, A. (2002). Avaliação de habilidades sociais de crianças com um inventário multimídia: indicadores sociométricos associados à freqüência versus dificuldade. Psicologia em estudo, 7(1), 61-73.

${ }^{*}$ Del Prette, Z. A. P. y Del Prette, A. (2003a). Habilidades sociais e dificuldades de aprendizagem: teoria e pesquisa sob um enfoque multimodal. En A. Del Prette y Z. A. P. Del Prette (Eds.), Habilidades sociais, desenvolvimento e aprendizagem: questões conceituais, avaliação e intervenção (pp. 167-206). Campinas: Alínea.

Del Prette, Z. A. P. y Del Prette, A. (2003b). No contexto da travessia para o ambiente de trabalho: treinamento de habilidades sociais com universitários. Estudos de Psicología, 8(3), 413420.

*Del Prette, Z. A. P. y Del Prette, A. (2009). Inventário de Habilidades Sociais para Adolescentes (IHSA-Del-Prette): Manual para aplicação, apuração e interpretação. São Paulo: Casa do Psicólogo.

Del Prette, Z. A. P., y Del Prette, A. (2010). Habilidades sociais e análise do comportamento: proximidade histórica e atualidades. Revista Perspectivas, 1(2), 104-115.

Del Prette, Z. A. P., y Del Prette, A. (2011). Psicologia das habilidades sociais: diversidade teórica e suas implicações (2da Ed.). Petrópolis: Vozes.

Delevati, D. M., Lima, D. V. B., Milka, L. y Silva, V. S. F. A. (2013). Estudo das habilidades sociais em pais de filhos internados por uso abusivo de substâncias psicoativas. Caderno de GraduaçãoCiências Biológicas e da Saúde-FITS, 1(2), 53-60.

*Dias, T. P., Freitas, L. C., Del Prette, Z. A. P. y Del Prette, A. (2011). Validação da escala de comportamentos sociais de préescolares para o Brasil. Psicologia em Estudo, 16(3), 447-457.

Feitosa, F. B., Del Prette, Z. A. P. y Del Prette, A. (2012). Habilidades sociais e rendimento acadêmico: a função mediadora da competência cognitiva. Temas em Psicologia, 20(1), 61-70.

Fernández Ballesteros, R. (2000). Introducción a la evaluación psicológica. Madrid: Pirámide.

*Flores, M. y Díaz-Loving, R. (2004). Escala Multidimensional de Asertividad (EMA). México, D.F.-Bogotá: Manual Moderno.

Gantman, A., Kapp, S. K., Orenski, K. y Laugeson, E. A. (2012). Social skills training for young adults with high-functioning autism spectrum disorders: A randomized controlled pilot study. Journal of autism and developmental disorders, 42(6), 1094-1103. doi: 10.1007/s10803-011-1350-6

Goleman, D. (1998). Working with emotional intelligence. New York: Bantam.
Gresham, F. M. (2011). Análise do comportamento aplicada às habilidades sociais. En A. Del Prette y Z. A. P. Del Prette (Eds.), Psicologia das habilidades sociais: diversidade teórica e suas implicações (2da Ed.) (pp. 17-66). Petrópolis: Vozes.

Gresham, F. M. y Elliot, S. (1984). Assessment and classification of children's social skills: A review of methods and issues. School Psychology Review, 13, 292-301.

Gresham, F. M., Vance, M. J., Chenier, J. y Hunter, K. (2013). Assessment and treatment of deficits in social skills functioning and social anxiety in children engaging in school refusal behaviors. En D. McKay y E. A. Storch (Eds.). Handbook of assessing variants and complications in anxiety disorders (pp. 15-28). New York: Springer.

Herrera Lestussi A., Freytes M. V., López G. E. y Olaz, F. O. (2012). Habilidades sociales en estudiantes universitarios de psicología. Un estudio comparativo. International Journal of Psychology and Psychological Therapy, 12(2), 277-287.

Inglés, C. J., Méndez, F. X., Hidalgo, M. D., Rosa, A. I. y Estévez, C. (2003). Evaluación de las habilidades sociales en educación secundaria: revisión de cuestionarios, inventarios y escalas. Psicología Educativa, 9(2), 71-87.

*Ipiña, M. J., Molina, L. y Reyna, C. (2010). Estructura factorial y consistencia interna de la escala MESSY (versión docente) en una muestra de niños argentinos. Suma Psicológica, 17(2) 151161.

*Ipiña, M. J., Molina, L. y Reyna, C. (2011). Propiedades psicométricas de la Escala MESSY (versión autoinforme) en niños argentinos. Revista de Psicología (PUCP), 29(2), 245-264.

${ }^{\star}$ Lacunza, A., Castro Solano, A. y Contini de González, N. (2009). Habilidades sociales preescolares: una escala para niños en contextos de pobreza. Revista de Psicología (PUCP), 27, 3-25.

${ }^{*}$ León, H. R. (2010). Modelos de prevención de trastornos de la conducta alimentaria: técnicas asertivas/habilidades sociales $y$ psicoeducativas (Tesis doctoral inédita). Facultad de Psicología, Universidad Nacional Autónoma de México, México.

León Rubio, J.M., León-Pérez, J.M., Herrera Sánchez, I.M. y De La Corte De La Corte, C. (2013). Entrenamiento en habilidades sociales y responsabilidad social corporativa: estudio de un caso. Apuntes de Psicología, 31(1), 101-107.

López López, P. (1996). Introducción a la Bibliometría. Valencia: Promolibro.

${ }^{*}$ Madrigala, M. L. y Halabíb, T. V. (2009). Validación y estandarización de la Escala de Asertividad de Rathus (RAS) en una muestra de adultos costarricenses. Revista Costarricense de Psicología, 28(41-42), 187-205.

Matson, J. L., Hattier, M. A. y Turygin, N. (2012). An evaluation of social skills in adults with pica, autism spectrum disorders, and intellectual disability. Journal of Developmental and Physical Disabilities, 24(5), 505-514. doi: 10.1007/s10882-012-9286-0

Mayer, J. D., Salovey, P. y Caruso, D. (2000). Models of emotional intelligence. En R. J. Sternberg (Ed.), Handbook of human intelligence (pp. 396-420). New York: Cambridge University Press. 
Miers, A. C., Blöte, A. W. y Westenberg, P. M. (2010). Peer perceptions of social skills in socially anxious and nonanxious adolescents. Journal of abnormal child psychology, 38(1), 33-41. doi: 10.1007/s10802-009-9345-x

Milsom, A. y Glanville, J. L. (2010). Factors mediating the relationship between social skills and academic grades in a sample of students diagnosed with learning disabilities or emotional disturbance. Remedial and Special Education, 31(4), 241-251. doi: 10.1177/0741932508327460

Montero, I. y León, O. G. (2007). Guía para nombrar los estudios de investigación en Psicología. International Journal of Clinical and Health Psychology, 7(3), 847-862.

${ }^{*}$ Moran, V., García Terán, M., Olaz, F. O. y Del Prette, Z. A. P. (2011). Construcción de la Versión Argentina del Inventario de Habilidades Sociales (IHS- AR). En M. J. F. Xavier Ribeiro, M. S. R. Tadeucci, E. A. S. de Araujo y Z. A. P. Del Prette (Eds.), Anais do $3^{\circ}$ Seminário Internacional de Habilidades Sociais (pp. 58). Taubate: UNITAU.

Olaz, F. O. (2011). Contribuções da teoría social cognitiva de Bandura para o treinamento de habilidades sociais. En A. Del Prette y Z. A. P. Del Prette (Orgs.), Psicologia das habilidades sociais: Diversidade teórica e suas implicações (2da Ed.) (pp. 109-148). Petrópolis: Vozes.

${ }^{*}$ Olaz, F. O. (2012). Escala de autoeficacia social para universitarios (EAS-U) Estudios Psicométricos y Bases Conceptuales. Saarbrücken: Editorial Académica Española.

Olaz, F. O., Cabanillas, G. A. y Medrano, L. (2011) Programas vivenciais versus instrucionais de habilidades sociais: impacto sobre a auto-eficácia social de universitários. En A. Del Prette y Z. A. P. Del Prette (Eds.), Habilidades sociais: Intervenções efetivas em grupo (pp. 175-202). São Paulo: Casa do Psicólogo.

*Olaz, F. O., Medrano, L., Greco, M. E. y Del Prette, Z. A. P. (2009). Argentinean adaptation of the social skills inventory IHS-DelPrette. The Spanish journal of psychology, 12(2), 756-766. doi: $10.1017 /$ S1138741600002122

${ }^{*}$ Pacheco, J. T., Teixeira, M. A. y Gomes, W. B. (1999). Estilos parentais e desenvolvimento de habilidades sociais na adolescência. Psicologia: Teoria e pesquisa, 15(2), 117-126.

Parault, S. J., Davis, H. A. y Pellegrini, A. D. (2007). The social contexts of bullying and victimization. The Journal of Early Adolescence, 27(2), 145-174. doi: 10.1177/0272431606294831

*Pasquali, L. y Gouveia, V. V. (1990). Escala de assertividade RathusRAS: Adaptação brasileira. Psicologia: Teoria e Pesquisa, 6(3), 233-249.

Penn, D. L., Kohlmaier, J. R. y Corrigan, P. W. (2000). Interpersonal factors contributing to the stigma of schizophrenia: Social skills, perceived attractiveness, and symptoms. Schizophrenia research, 45(1), 37-45. doi: 10.1016/S0920-9964(99)00213-3

${ }^{*}$ Pizzinato, A. y Sarriera, J. C. (2003). Competência social infantil: análise discriminante entre crianças imigrantes e não imigrantes no contexto escolar de Porto Alegre. Psicologia em Estudo, 8(2), 115-122.
Prytz Nilsson, N. y Suárez, A. B. (2009). Estudio bibliométrico de las publicaciones científicas sobre el área de Habilidades Sociales en América Latina (Tesis de licenciatura inédita). Facultad de Psicología. Universidad Nacional de Córdoba. Argentina.

Rasmussen, C., Becker, M., McLennan, J., Urichuk, L. y Andrew, G. (2011). An evaluation of social skills in children with and without prenatal alcohol exposure. Child: Care, Health and Development, 37, 711-718. doi:10.1111/j.13652214.2010.01152.x

${ }^{*}$ Reyna, C. y Brussino, S. (2009). Propiedades psicométricas de la escala de comportamiento preescolar y jardín infantil en una muestra de niños argentinos de 3 a 7 años. Psykhe, 18(2), 127140.

Reyna, C. y Brussino, S. (2011). Avaliação de habilidades sociais de crianças na América Latina. Psicologia em Estudo, 16(3), 359367.

Richaud, M. C. (2005). Desarrollos del Análisis Factorial para el estudio de items dicotómicos y ordinales. Interdisciplinaria, 22(2), 237-251.

Rom, M. y Silvestre, N. (2012). Las relaciones sociales entre adolescentes sordos y sus compañeros de clase oyentes. Infancia y Aprendizaje, 35(1), 5-22.

Rueda-Clausen Gómez, C. F., Villa-Roel Gutíerrez, C. y RuedaClausen Pinzón, C. E. (2010). Indicadores bibliométricos: origen, aplicación, contradicción y nuevas propuestas. Medunab, 8(1), 29-36.

Russell, J. M. (2004). Obtención de indicadores bibliométricos a partir de la utilización de las herramientas tradicionales de información. Recuperado de http://www.eventos.bvsalud.org/ INFO2004/docs/es/RussellJM.pdf.

Saldaña, M. E. G. y Cruz, R. G. (2013). Programa de entrenamiento en habilidades sociales, en adolescentes con problemas de conducta. Revista Científico Electrónica de Psicología, 1(1), 8-14.

Smith, K. R. y Matson, J. L. (2010). Social skills: Differences among adults with intellectual disabilities, co-morbid autism spectrum disorders and epilepsy. Research in Developmental Disabilities, 31(6), 1366-1372. doi: 10.1016/j.ridd.2010.07.002

*Teodoro M. L. M., Käppler, K. C., Rodrigues, J. L., de Freitas, P. M. y Haase, V. G. (2005). The Matson Evaluation of Social Skills with Youngsters (MESSY) and its adaptation for Brazilian children and adolescents. Interamerican Journal of Psychology, 39(2), 239-246.

Tornimbeni, S., Pérez, E. y Olaz, F. (2008). Introducción a la psicometría. Buenos Aires: Paidós.

Troisi, A., Spalletta, G. y Pasini, A. (1998). Non-verbal behaviour deficits in schizophrenia: an ethological study of drug-free patients. Acta Psychiatrica Scandinavica, 97(2), 109-115.

*Velázquez Altamirano, M., Arellanez Hernández, J. L. y Díaz Negrete, D. B. (2009). Prueba de instrumentos para la medición de ansiedad y asertividad en usuarios de sustancias. México: CIJ. 
*Villa, M. B. (2005). Habilidades sociais no casamento: Avaliação e contribuição para a satisfação conjugal (Tesis doctoral, Programa de Pós-Graduação em Psicologia, Universidad de
Sao Paulo, Ribeirão Preto, Brasil). Recuperada de http://www. teses.usp.br/teses/disponiveis/59/59137/tde-22012008-83741/ publico/TeseMiriam.pdf 